\title{
Occupational health status of High School teachers in Tirur educational District ws.r. to musculoskeletal disorders -A Cross sectional study
}

\author{
Research Article
}

\section{Rajarajeswary $\mathbf{T} \mathbf{R}^{1 *}$, Surendran E2, Sowmya C Nair ${ }^{3}$}

\author{
1. Post Graduate scholar, 2. Associate Professor, 3. Assistant Professor
}

Department of Swasthavritta, V P S V Ayurveda College, Kottakkal, Kerala.

\begin{abstract}
A cross sectional study was carried out among 140 high school teachers selected from 4 sub educational districts of Tirur educational district through multistage sampling. Both sexes were included in the study. The tool used for the study was Dutch musculoskeletal questionnaire and filled up by personal interview. The data obtained was statistically analyzed by using Chi square test, Yates correction and Fisher's exact test. Most reported musculoskeletal complaints were knee pain (43\%), shoulder pain (34\%), Lower back pain (23\%) and ankle pain (22\%). This study shows that musculoskeletal disorders are prevalent among high school teachers of Tirur educational district. Musculoskeletal disorders are associated with factors such as age, gender and occupational factors such as prolonged standing and overtime work.
\end{abstract}

Key Words: High school teachers, musculoskeletal disorders, occupational health, government school and aided schools, Kerala.

\section{Introduction}

The prevention of disease and maintenance of highest degree of physical, mental and social welfare of workers in all occupations is the aim of preventive medicine and occupational health.(1) The World Health Organization considers work place as one of the priority settings for health promotion because it influences physical, mental, economical and social well-being and offers an ideal setting and infrastructure to support the promotion of health of a large audience. (2) Just like home, the place of work is also an important environment for an earning person. Such a person spends nearly 6 to 8 hours a day in the working place till the retirement for about 3 decades. The worker as well as the working environment should be healthy, safe and free from harmful agents.It is becoming more complicated as man is more ingenious because of industrialization and urbanization.(3)

Work related musculoskeletal disorders are one among the occupational health problem. The work of a teacher does not only involve teaching students but also preparing lessons, assessing students' work and also

* Corresponding Author:

Rajarajeswary TR

Department of Swasthavritta,

V P S V Ayurveda College,

Kottakkal, Kerala - 676501 .

Email id - rajarajeswarytr@gmail.com being involved in extracurricular activities such as sports. Teachers also participate in different school committees. In some areas, teaching is done under unfavourable circumstances, in which teachers must mobilize their physical, cognitive and affective capability to reach a teaching production objective, over demanding or generating effort to their psychophysiology functions.(4) In our state educational issues such as working stress, reduction in size and number of classes have already drawn much public attention. However, very little attention has been given to somatic health problems of teachers. So there is a need to study the health problems among school teachers. This study is giving focus to musculoskeletal disorders among the high school teachers.

\section{Materials \& Methods}

A cross sectional survey conducted among teachers of Tirur educational district. Teachers were selected according to the inclusion criteria and who were willing to give written consent. A pamphlet in Malayalam language containing the details of the research was given to the participants. Consent form in Malayalam language was prepared and prior consent of all the participants were obtained on the consent form. They were given the freedom to quit from the study at any part of it at their own will.

\section{Inclusion criteria}

- High school teachers from government and aided schools 
- At least 6 years of teaching experience

- 30- 60 years of age group from both sexes

\section{Exclusion criteria}

- Temporary staff

- Staff who had taken more than one year leave

- Teachers working in un-aided schools

The study was descriptive cross sectional study among 140 samples.

\section{Sampling}

Multi stage sampling was used in the study. The target population of this study was high school teachers of Kerala; from that group high school teachers of Tirur educational district selected as source of population. In Tirur educational district there are 7 educational sub districts. From these 4 educational sub district were selected by simple random sampling method. From each sub district 2 schools ( 1 government and 1 aided) was selected by purposive sampling method.

Teachers from each school were selected based on inclusion criteria and availability of teachers.

Table No. 1 Educational subdistricts \& distribution of teachers

\begin{tabular}{|c|c|c|}
\hline Edu-subdistricts & No.of teacher & Percentage \\
\hline Tirur & 46 & $33 \%$ \\
Kuttipuram & 32 & $23 \%$ \\
Edappal & 32 & $23 \%$ \\
Ponnani & 30 & $21 \%$ \\
\hline
\end{tabular}

\section{Assessment tools}

1. Case record form - To collect personal data, history of illness, family history , occupational history

2. Dutch musculoskeletal questionnaire shortened version (5) contains
- Background variables (e.g. age, gender, education, duration of employment,

- work history, shift work);

- Tasks (prevalence rates and perceived heaviness of task demands);

- 9 sub domains (Musculoskeletal workload of different body parts)

- Work pace and psychosocial working conditions (demands, control and autonomy, work organization and social support, work satisfaction)

In the present study 9 subdomains were analysed. Pain/ discomfort on neck, upper-back lower back, shoulder, elbow, wrist/hands, hips, knees, ankles/ feet were statistically tested.

The data were collected by personal interview method from available samples at the time of study.The statistical analysis of data was carried out with the help of Statistical

Package for Social Sciences (SPSS) 21.0 version

\section{Results \& Discussion}

Statistical analysis of the current study is done to find out the association between the musculoskeletal disorders prevalent in high school teachers and the risk factors present in the same occupation.

To find out the association Chi square test is applied.

The test is done to find out whether any association exists between

1. Musculoskeletal disorders and personal factors

2. Musculoskeletal disorders and occupational factors among high school teachers in Tirur educational district.

When the expected frequency is less than 5, Yates correction has been done. When the observed frequency is less than 5, Fisher's exact test is applied.

1.Association between age and musculoskeletal disorder Table No.2 Association of age \& MSD

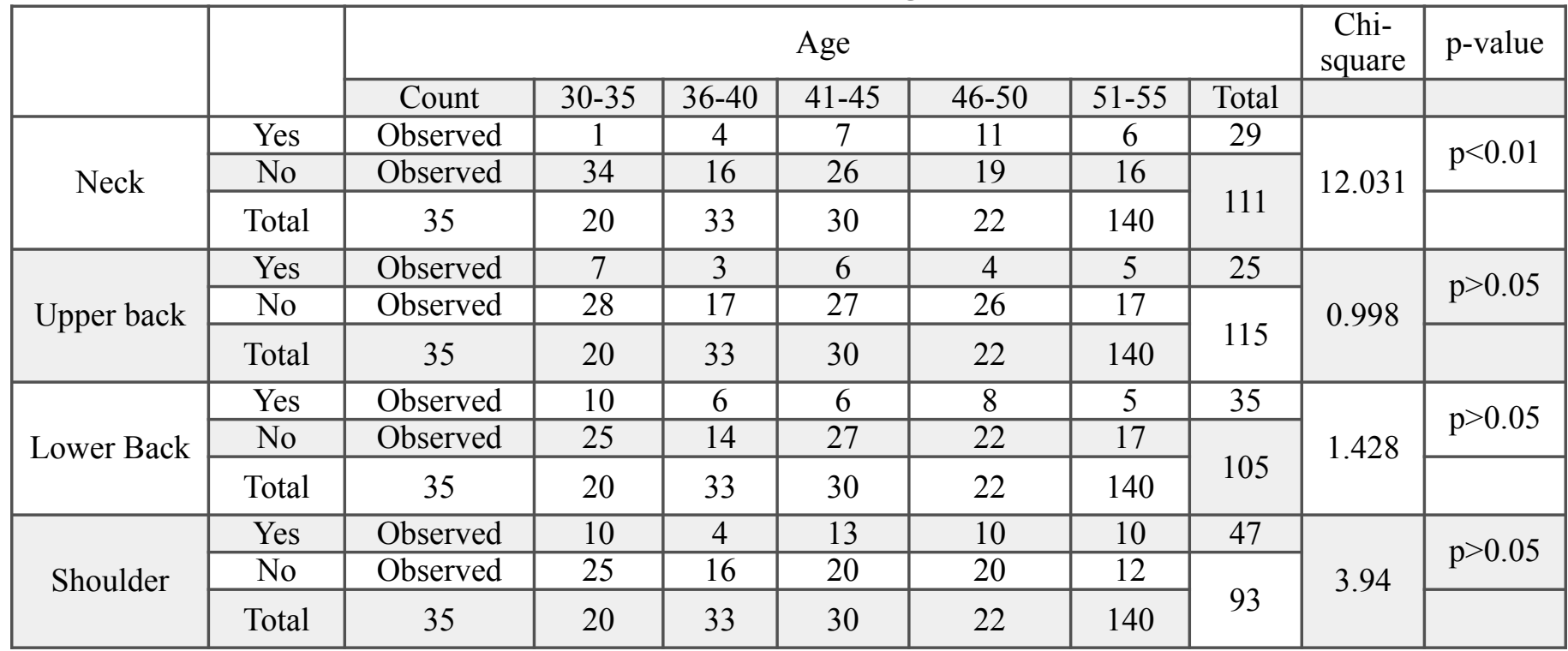


International Journal of Ayurvedic Medicine, Vol 11 (1), 98-107

\begin{tabular}{|c|c|c|c|c|c|c|c|c|c|c|}
\hline \multirow{3}{*}{ Elbows } & Yes & Observed & 4 & 1 & 1 & 4 & 4 & 14 & \multirow{3}{*}{4.423} & \multirow{2}{*}{$\mathrm{p}>0.05$} \\
\hline & No & Observed & 31 & 19 & 32 & 26 & 18 & \multirow{2}{*}{126} & & \\
\hline & Total & 35 & 20 & 33 & 30 & 22 & 140 & & & \\
\hline \multirow{3}{*}{$\begin{array}{l}\text { Wrists/ } \\
\text { hands }\end{array}$} & Yes & Observed & 1 & 0 & 1 & 4 & 4 & 10 & \multirow{3}{*}{9.124} & \multirow{2}{*}{$p>0.05$} \\
\hline & No & Observed & 34 & 20 & 32 & 26 & 18 & \multirow{2}{*}{130} & & \\
\hline & Total & 35 & 20 & 33 & 30 & 22 & 140 & & & \\
\hline \multirow{3}{*}{ Hips/ } & Yes & Observed & 0 & 1 & 5 & 5 & 2 & 13 & \multirow{3}{*}{7.308} & \multirow{2}{*}{$p>0.05$} \\
\hline & No & Observed & 35 & 19 & 28 & 25 & 20 & \multirow{2}{*}{127} & & \\
\hline & Total & 35 & 20 & 33 & 30 & 22 & 140 & & & \\
\hline \multirow{3}{*}{ Knees } & Yes & Observed & 15 & 10 & 13 & 11 & 15 & 64 & \multirow{3}{*}{6.259} & \multirow{2}{*}{$p>0.05$} \\
\hline & No & Observed & 20 & 10 & 20 & 19 & 7 & \multirow{2}{*}{76} & & \\
\hline & Total & 35 & 20 & 33 & 30 & 22 & 140 & & & \\
\hline \multirow{3}{*}{ Ankles/Feet } & Yes & Observed & 1 & 2 & 8 & 10 & 10 & 31 & \multirow{3}{*}{18.46} & \multirow{2}{*}{$\mathrm{p}<0.01$} \\
\hline & No & Observed & 34 & 18 & 25 & 20 & 12 & \multirow{2}{*}{109} & & \\
\hline & Total & 35 & 20 & 33 & 30 & 22 & 140 & & & \\
\hline
\end{tabular}

The mean age in the present study is 42.22 . In this study neck pain and ankle pain are significantly associated with age. Majority (36.7\%) of teachers who reported neck pain comes under the age group 46-50.27.3\% comes under age group 51-55. $21.2 \%$ fall into the age group of 41-45. Study by Erick and D.smith states a positive association between increasing age and the development of MSD. These studies found that teachers aged 40 years or over were more likely to report MSD of different body sites. It has been suggested that the likely reason for a higher prevalence of MSD among older teachers is that as people age, there is a gradual decline in muscle mass and they lose connective tissue elasticity and undergo a thinning of the cartilage between joints.

Apart from natural wear and tear on the body, MSD among older teachers may also be influenced by work environment and the organisation of work. (6)

\section{Association between Gender and musculoskeletal disorder \\ Table No. 3 Association of gender with MSD}

\begin{tabular}{|c|c|c|c|c|c|c|c|}
\hline & & \multicolumn{4}{|c|}{ Sex } & Chi-square & $\mathrm{p}$-value \\
\hline & & Count & Male & Female & Total & & \\
\hline \multirow{3}{*}{ Neck } & Yes & Observed & 14 & 15 & 29 & \multirow{3}{*}{0.097} & \multirow{2}{*}{$\mathrm{p}>0.05$} \\
\hline & No & Observed & 50 & 61 & & & \\
\hline & Total & 64 & 76 & 140 & 111 & & \\
\hline \multirow{4}{*}{ Upper back } & Y & Observed & 9 & 16 & 25 & \multirow{4}{*}{1.157} & \multirow{3}{*}{$\mathrm{p}>0.05$} \\
\hline & res & Percentage & $14.1 \%$ & $21.1 \%$ & $17.9 \%$ & & \\
\hline & No & Observed & 55 & 60 & \multirow{2}{*}{115} & & \\
\hline & Total & 64 & 76 & 140 & & & \\
\hline \multirow{3}{*}{ Lower Back } & Yes & Observed & 15 & 20 & 35 & \multirow{3}{*}{0.154} & \multirow{2}{*}{$\mathrm{p}>0.05$} \\
\hline & No & Observed & 49 & 56 & 105 & & \\
\hline & Total & 64 & 76 & 140 & 105 & & \\
\hline \multirow{3}{*}{ Shoulder } & Yes & Observed & 18 & 29 & 47 & \multirow{3}{*}{1.568} & \multirow{2}{*}{$\mathrm{p}>0.05$} \\
\hline & No & Observed & 46 & 47 & & & \\
\hline & Total & 64 & 76 & 140 & 95 & & \\
\hline \multirow{3}{*}{ Elbows } & Yes & Observed & 4 & 10 & 14 & \multirow{3}{*}{1.842} & \multirow{2}{*}{$p>0.05$} \\
\hline & No & Observed & 60 & 66 & & & \\
\hline & Total & 64 & 76 & 140 & 120 & & \\
\hline \multirow{3}{*}{$\begin{array}{l}\text { Wrists/ } \\
\text { hands }\end{array}$} & Yes & Observed & 1 & 9 & 10 & \multirow{3}{*}{5.535} & \multirow{2}{*}{$\mathrm{p}<0.05$} \\
\hline & No & Observed & 63 & 67 & 120 & & \\
\hline & Total & 64 & 76 & 140 & 150 & & \\
\hline \multirow{3}{*}{ Hips/ } & Yes & Observed & 5 & 8 & 13 & \multirow{3}{*}{0.304} & \multirow{3}{*}{$\mathrm{p}>0.05$} \\
\hline & No & Observed & 59 & 68 & \multirow{2}{*}{127} & & \\
\hline & Total & 64 & 76 & 140 & & & \\
\hline
\end{tabular}


Rajarajeswary TR et.al., Musculoskeletal disorders in high school teachers of Tirur educational district-A cross sectional study

\begin{tabular}{|c|c|c|c|c|c|c|c|}
\hline \multirow{3}{*}{ Knees } & Yes & Observed & 24 & 40 & 64 & \multirow{2}{*}{3.206} & $\mathrm{p}>0.05$ \\
\cline { 2 - 5 } & No & Observed & 40 & 36 & 76 & \\
\cline { 2 - 5 } & Total & 64 & 76 & 140 & & \\
\hline \multirow{3}{*}{ Ankles/Feet } & Yes & Observed & 8 & 23 & 31 & \multirow{2}{*}{6.359} & $\mathrm{p}<0.05$ \\
\cline { 2 - 5 } & No & Observed & 56 & 53 & 109 & \\
\cline { 2 - 5 } & Total & 64 & 76 & 140 & & \\
\hline
\end{tabular}

In the current study neck pain is more reported by male participants $(21.9 \%)$ and female participants of 19.7\%. Musculoskeletal disorders of other body sites are more reported by the female teachers than male participants. There is statistically significant association shown by wrist pain and ankle joint pain with female gender $(\mathrm{p}<0.05)$. In a Chinese study, musculoskeletal complaints are often associated with the female gender. $93.5 \%$ women complained of musculoskeletal problems while $83.0 \%$ male teachers reported the same $;(p<.001)$. Females, representing a higher proportion of teachers often have a higher prevalence of MSD. This study also found that women bore more heavy housework responsibilities than men in daily life. Differences in household task participation may also explain musculoskeletal differences.(7) In the present study musculoskeletal disorders in neck is more reported by male gender which is inconsistent with other study.

3.Association between addiction and musculoskeletal disorders

Table No.4 Association of addiction and musculoskeletal disorders

\begin{tabular}{|c|c|c|c|c|c|c|c|}
\hline & & \multicolumn{4}{|c|}{ Addiction } & Chi-square & p-value \\
\hline & & Count & Smoking & None & Total & & \\
\hline \multirow{3}{*}{ Neck } & Yes & Observed & 2 & 27 & 29 & \multirow{3}{*}{0.608} & \multirow{2}{*}{$\mathrm{p}>0.05$} \\
\hline & No & Observed & 4 & 107 & \multirow{2}{*}{111} & & \\
\hline & Total & 6 & 134 & 140 & & & \\
\hline \multirow{3}{*}{ Upper back } & Yes & Observed & 1 & 24 & 25 & \multirow{3}{*}{0.006} & \multirow{2}{*}{$p>0.05$} \\
\hline & No & Observed & 5 & 110 & \multirow{2}{*}{115} & & \\
\hline & Total & 6 & 134 & 140 & & & \\
\hline \multirow{4}{*}{ Lower Back } & Yes & Observed & 1 & 34 & 35 & \multirow{4}{*}{0.232} & \multirow{3}{*}{$\mathrm{p}>0.05$} \\
\hline & $N$ & Observed & 5 & 100 & 105 & & \\
\hline & No & Percentage & 83.3 & 74.6 & & & \\
\hline & Total & 6 & 134 & 140 & 15 & & \\
\hline \multirow{3}{*}{ Shoulder } & Yes & Observed & 1 & 46 & 47 & \multirow{3}{*}{0.803} & \multirow{2}{*}{$\mathrm{p}>0.05$} \\
\hline & No & Observed & 5 & 88 & \multirow{2}{*}{93} & & \\
\hline & Total & 6 & 134 & 140 & & & \\
\hline \multirow{3}{*}{ Elbows } & Yes & Observed & 1 & 13 & 14 & \multirow{3}{*}{0.31} & \multirow{2}{*}{$\mathrm{p}>0.05$} \\
\hline & No & Observed & 5 & 121 & & & \\
\hline & Total & 6 & 134 & 140 & 126 & & \\
\hline \multirow{3}{*}{ Wrists/hands } & Yes & Observed & 0 & 10 & 10 & \multirow{3}{*}{0.482} & \multirow{2}{*}{$\mathrm{p}>0.05$} \\
\hline & No & Observed & 6 & 124 & & & \\
\hline & Total & 6 & 134 & 140 & 150 & & \\
\hline \multirow{3}{*}{ Hips/ } & Yes & Observed & 0 & 13 & 13 & \multirow{3}{*}{0.642} & \multirow{2}{*}{$\mathrm{p}>0.05$} \\
\hline & No & Observed & 6 & 121 & & & \\
\hline & Total & 6 & 134 & 140 & 127 & & \\
\hline \multirow{3}{*}{ Knees } & Yes & Observed & 3 & 61 & 64 & \multirow{3}{*}{0.046} & \multirow{2}{*}{$p>0.05$} \\
\hline & No & Observed & 3 & 73 & & & \\
\hline & Total & 6 & 134 & 140 & 16 & & \\
\hline \multirow{3}{*}{ Ankles/Feet } & Yes & Observed & 0 & 31 & 31 & & $\mathrm{n}>0005$ \\
\hline & No & Observed & 6 & 103 & \multirow{2}{*}{109} & 1.783 & $P=0.00$ \\
\hline & Total & 6 & 134 & 140 & & & \\
\hline
\end{tabular}

In the present study there is no statistically significant association between addiction and musculoskeletal disorders. 
International Journal of Ayurvedic Medicine, Vol 11 (1), 98-107

4.Association between work experience and Musculoskeletal disorder

Table No.5 Work experience and musculoskeletal disorders

\begin{tabular}{|c|c|c|c|c|c|c|c|c|c|}
\hline & & \multicolumn{6}{|c|}{ Work Experience } & Chi-square & p-value \\
\hline & & Count & 6 to 12 & 13 to 19 & 20 to 26 & 27 to 33 & Total & & \\
\hline \multirow{3}{*}{ Neck } & Yes & Observed & 7 & 7 & 11 & 4 & 29 & \multirow{3}{*}{6.644} & \multirow[b]{2}{*}{$p>0.05$} \\
\hline & No & Observed & 56 & 19 & 28 & 8 & \multirow{2}{*}{111} & & \\
\hline & Total & 63 & 26 & 39 & 12 & 140 & & & \\
\hline \multirow{3}{*}{$\begin{array}{l}\text { Upper } \\
\text { back }\end{array}$} & Yes & Observed & 11 & 6 & 5 & 3 & 25 & \multirow{3}{*}{1.582} & \multirow{2}{*}{$p>0.05$} \\
\hline & No & Observed & 52 & 20 & 34 & 9 & \multirow{2}{*}{115} & & \\
\hline & Total & 63 & 26 & 39 & 12 & 140 & & & \\
\hline \multirow{3}{*}{$\begin{array}{l}\text { Lower } \\
\text { Back }\end{array}$} & Yes & Observed & 16 & 7 & 8 & 4 & 35 & \multirow{3}{*}{0.92} & $n>0,05$ \\
\hline & No & Observed & 47 & 19 & 31 & 8 & \multirow{2}{*}{105} & & $p>0.05$ \\
\hline & Total & 63 & 26 & 39 & 12 & 140 & & & \\
\hline \multirow{3}{*}{ Shoulder } & Yes & Observed & 18 & 10 & 12 & 7 & 47 & \multirow{3}{*}{4.422} & $n>0,05$ \\
\hline & No & Observed & 45 & 16 & 27 & 5 & \multirow{2}{*}{93} & & $p>0.05$ \\
\hline & Total & 63 & 26 & 39 & 12 & 140 & & & \\
\hline \multirow{3}{*}{ Elbows } & Yes & Observed & 5 & 2 & 4 & 3 & 14 & \multirow{3}{*}{3.455} & $n>0,05$ \\
\hline & No & Observed & 58 & 24 & 35 & 9 & \multirow{2}{*}{126} & & $p>0.03$ \\
\hline & Total & 63 & 26 & 39 & 12 & 140 & & & \\
\hline \multirow{3}{*}{$\begin{array}{l}\text { Wrists/ } \\
\text { hands }\end{array}$} & Yes & Observed & 1 & 2 & 4 & 3 & 10 & \multirow{3}{*}{9.283} & $\mathrm{n}<0,01$ \\
\hline & No & Observed & 62 & 24 & 35 & 9 & \multirow{2}{*}{130} & & $p<0.01$ \\
\hline & Total & 63 & 26 & 39 & 12 & 140 & & & \\
\hline \multirow{3}{*}{ Hips/ } & Yes & Observed & 1 & 4 & 7 & 1 & 13 & \multirow{3}{*}{$9.068^{*}$} & $\mathrm{n}<0,01$ \\
\hline & No & Observed & 62 & 22 & 32 & 11 & \multirow{2}{*}{127} & & $p<0.01$ \\
\hline & Total & 63 & 26 & 39 & 12 & 140 & & & \\
\hline \multirow{3}{*}{ Knees } & Yes & Observed & 27 & 13 & 15 & 9 & 64 & \multirow{3}{*}{5.374} & $n>0,05$ \\
\hline & No & Observed & 36 & 13 & 24 & 3 & \multirow{2}{*}{76} & & $p>0.05$ \\
\hline & Total & 63 & 26 & 39 & 12 & 140 & & & \\
\hline \multirow{3}{*}{$\begin{array}{c}\text { Ankles/ } \\
\text { Feet }\end{array}$} & Yes & Observed & 4 & 11 & 12 & 4 & 31 & \multirow{3}{*}{17.803} & $n>0,05$ \\
\hline & No & Observed & 59 & 15 & 27 & 8 & \multirow{2}{*}{109} & & $p=0.0 J$ \\
\hline & Total & 63 & 26 & 39 & 12 & 140 & & & \\
\hline
\end{tabular}

Musculoskeletal disorders in hip region and wrists show statistically significant association with work experience $(\mathrm{p}<0.01) .25 \%$ teachers who reported wrist pain have work experience ranging from 27-33 years. $10.3 \%$ teachers with wrist pain have an experience 20-26 years. 7.7\% teachers come under 13-19 years of experience and only $1.9 \%$ teacher comes under 6-12 years of experience. In the case of hip pain $17.9 \%$ teachers have an experience of 20-26 years. $15.4 \%$ teachers with hip pain have an experience of 13-19 years. Only 8.3\% teachers with experience of 27-33 years reported hip joint pain. This is a contradictory finding between length of employment and development of musculoskeletal disorders. Longer length of employment is positively associated with wrist pain. This finding go hand in hand with the study by T.Mariammal et al done among teachers in thoothukudi town. It is stated that as the experience of teachers increased, the development of the disorders were also high. The much affected disorders observed were Neck and shoulder pain and pain in joints of legs and hands.(8) It can been interpreted that longer the exposure time to occupational risk factors higher the chance of getting job related disorders.

5.Association between working hour and Musculoskeletal disorder

Table No.6 Association of working hour and Musculoskeletal disorder

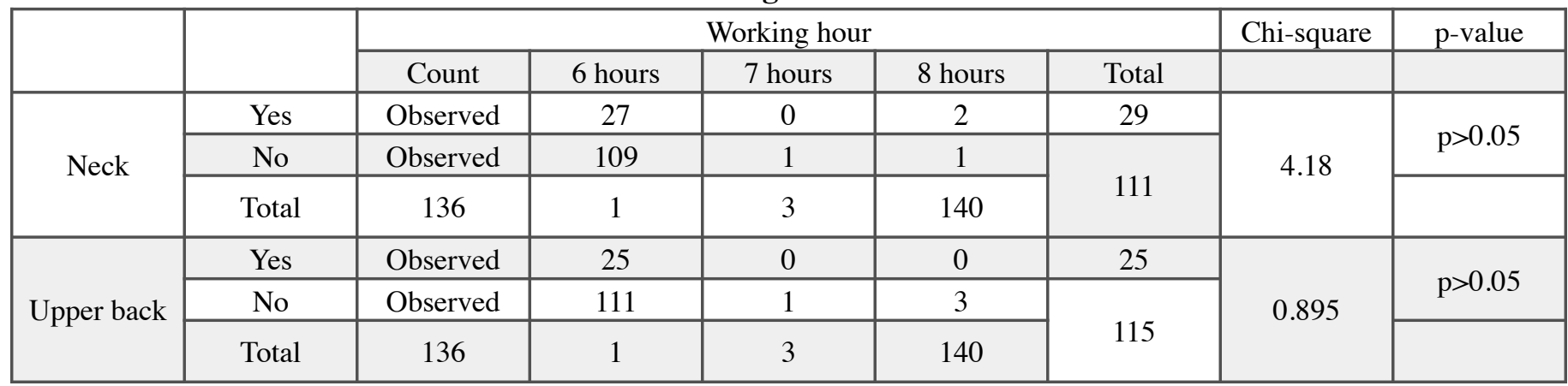


Rajarajeswary TR et.al., Musculoskeletal disorders in high school teachers of Tirur educational district-A cross sectional study

\begin{tabular}{|c|c|c|c|c|c|c|c|c|}
\hline \multirow{3}{*}{$\begin{array}{l}\text { Lower } \\
\text { Back }\end{array}$} & Yes & Observed & 33 & 0 & 2 & 35 & \multirow{3}{*}{3.15} & \multirow{2}{*}{$\mathrm{p}>0.05$} \\
\hline & No & Observed & 103 & 1 & 1 & \multirow{2}{*}{105} & & \\
\hline & Total & 136 & 1 & 3 & 140 & & & \\
\hline \multirow{3}{*}{ Shoulder } & Yes & Observed & 47 & 0 & 0 & 47 & \multirow{3}{*}{2.081} & \multirow{2}{*}{$\mathrm{p}>0.05$} \\
\hline & No & Observed & 89 & 1 & 3 & \multirow{2}{*}{93} & & \\
\hline & Total & 136 & 1 & 3 & 140 & & & \\
\hline \multirow{3}{*}{ Elbows } & Yes & Observed & 14 & 0 & 0 & 14 & \multirow{3}{*}{0.458} & \multirow{2}{*}{$\mathrm{p}>0.05$} \\
\hline & No & Observed & 122 & 1 & 3 & \multirow{2}{*}{126} & & \\
\hline & Total & 136 & 1 & 3 & 140 & & & \\
\hline \multirow{3}{*}{$\begin{array}{l}\text { Wrists/ } \\
\text { hands }\end{array}$} & Yes & Observed & 10 & 0 & 0 & 10 & \multirow{3}{*}{0.317} & \multirow{2}{*}{$\mathrm{p}>0.05$} \\
\hline & No & Observed & 126 & 1 & 3 & \multirow{2}{*}{130} & & \\
\hline & Total & 136 & 1 & 3 & 140 & & & \\
\hline \multirow{3}{*}{ Hips/ } & Yes & Observed & 13 & 0 & 0 & 13 & \multirow{3}{*}{0.421} & \multirow{2}{*}{$\mathrm{p}>0.05$} \\
\hline & No & Observed & 123 & 1 & 3 & \multirow{2}{*}{127} & & \\
\hline & Total & 136 & 1 & 3 & 140 & & & \\
\hline \multirow{3}{*}{ Knees } & Yes & Observed & 63 & 0 & 1 & 64 & \multirow{3}{*}{1.048} & \multirow{2}{*}{$\mathrm{p}>0.05$} \\
\hline & No & Observed & 73 & 1 & 2 & \multirow{2}{*}{76} & & \\
\hline & Total & 136 & 1 & 3 & 140 & & & \\
\hline \multirow{3}{*}{$\begin{array}{c}\text { Ankles/ } \\
\text { Feet }\end{array}$} & Yes & Observed & 30 & 0 & 1 & 31 & \multirow{3}{*}{0.503} & \multirow{2}{*}{$\mathrm{p}>0.05$} \\
\hline & No & Observed & 106 & 1 & 2 & \multirow{2}{*}{109} & & \\
\hline & Total & 136 & 1 & 3 & 140 & & & \\
\hline
\end{tabular}

There is no significant association with working hours musculoskeletal disorders in neck, shoulder, elbows, wrists, upper back, lower back, hip, knees, ankles and feet

6. Association between time of standing and Musculoskeletal disorder Table No.7 Time of standing and musculoskeletal disorders

\begin{tabular}{|c|c|c|c|c|c|c|c|c|}
\hline & & \multicolumn{5}{|c|}{ Time of standing } & \multirow[t]{2}{*}{ Chi-square } & \multirow[t]{2}{*}{ p-value } \\
\hline & & Count & $4-5 \mathrm{hrs}$ & 6-7 hrs & $8-9 \mathrm{hrs}$ & Total & & \\
\hline \multirow{3}{*}{ Neck } & Yes & Observed & 22 & 5 & 2 & 29 & \multirow{3}{*}{4.196} & \multirow{2}{*}{$\mathrm{p}>0.05$} \\
\hline & No & Observed & 94 & 16 & 1 & \multirow{2}{*}{111} & & \\
\hline & Total & 116 & 21 & 3 & 140 & & & \\
\hline \multirow{3}{*}{ Upper back } & Yes & Observed & 20 & 5 & 0 & 25 & \multirow{3}{*}{1.189} & \multirow{2}{*}{$\mathrm{p}>0.05$} \\
\hline & No & Observed & 96 & 16 & 3 & \multirow{2}{*}{115} & & \\
\hline & Total & 116 & 21 & 3 & 140 & & & \\
\hline \multirow{3}{*}{$\begin{array}{l}\text { Lower } \\
\text { Back }\end{array}$} & Yes & Observed & 28 & 6 & 1 & 35 & \multirow{3}{*}{0.3} & \multirow{2}{*}{$\mathrm{p}>0.05$} \\
\hline & No & Observed & 88 & 15 & 2 & \multirow{2}{*}{105} & & \\
\hline & Total & 116 & 21 & 3 & 140 & & & \\
\hline \multirow{3}{*}{ Shoulder } & Yes & Observed & 39 & 8 & 0 & 47 & \multirow{3}{*}{1.709} & \multirow{2}{*}{$\mathrm{p}>0.05$} \\
\hline & No & Observed & 77 & 13 & 3 & \multirow{2}{*}{93} & & \\
\hline & Total & 116 & 21 & 3 & 140 & & & \\
\hline \multirow{3}{*}{ Elbows } & Yes & Observed & 13 & 1 & 0 & 14 & \multirow{3}{*}{1.161} & \multirow{2}{*}{$\mathrm{p}>0.05$} \\
\hline & No & Observed & 103 & 20 & 3 & \multirow{2}{*}{126} & & \\
\hline & Total & 116 & 21 & 3 & 140 & & & \\
\hline \multirow{3}{*}{$\begin{array}{l}\text { Wrists/ } \\
\text { hands }\end{array}$} & Yes & Observed & 5 & 5 & 0 & 10 & \multirow{3}{*}{10.429} & \multirow{2}{*}{$\mathrm{p}<0.05$} \\
\hline & No & Observed & 111 & 16 & 3 & \multirow{2}{*}{130} & & \\
\hline & Total & 116 & 21 & 3 & 140 & & & \\
\hline \multirow{3}{*}{ Hips/ } & Yes & Observed & 12 & 1 & 0 & 13 & \multirow{3}{*}{0.972} & \multirow{2}{*}{$\mathrm{p}>0.05$} \\
\hline & No & Observed & 104 & 20 & 3 & \multirow{2}{*}{127} & & \\
\hline & Total & 116 & 21 & 3 & 140 & & & \\
\hline
\end{tabular}


International Journal of Ayurvedic Medicine, Vol 11 (1), 98-107

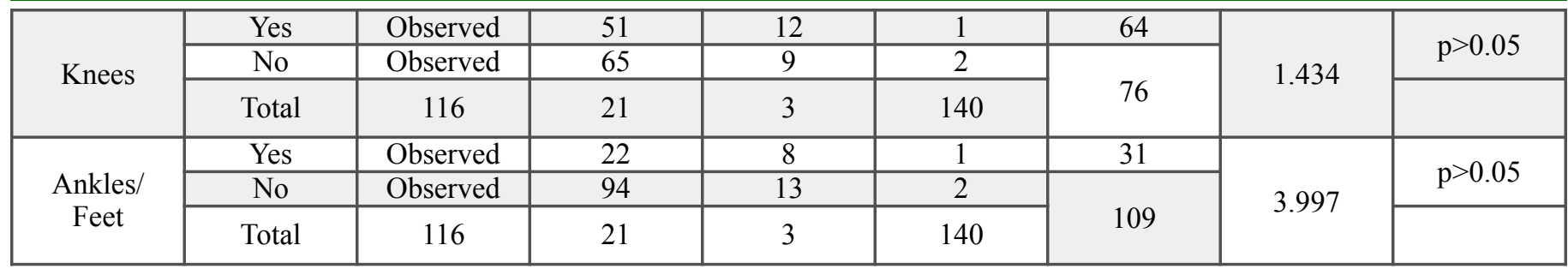

Wrist pain shows statistically significant association with time of standing and MSD $(\mathrm{p}<0.05)$. Musculoskeletal disorders in other body sites do not show any statistical significance. This is supported by another study by T.mariammal et al in which $66.59 \%$ reported pain in hands. Lifting of hands and head during writing in the black board may be the causative factor for neck and shoulder pain and also pain in hands and joints of hands.

7.Association between duration of computer use and musculoskeletal disorders Table No.8 Duration of computer use \& musculoskeletal disorders

\begin{tabular}{|c|c|c|c|c|c|c|c|c|c|c|}
\hline & & \multicolumn{7}{|c|}{ Duration of computer use (in hours) } & \multirow[t]{2}{*}{$\begin{array}{l}\text { Chi- } \\
\text { square }\end{array}$} & \multirow[t]{2}{*}{ p-value } \\
\hline & & count & $0 \mathrm{hr}$ & $\begin{array}{c}\text { Upto } 1 \\
\text { hr }\end{array}$ & $1-2 \mathrm{hr}$ & $2-3 \mathrm{hr}$ & $>3 \mathrm{hr}$ & Total & & \\
\hline \multirow{3}{*}{ Neck } & Yes & Observed & 12 & 10 & 3 & 3 & 1 & 29 & \multirow{3}{*}{3.136} & \multirow{2}{*}{$\mathrm{p}>0.05$} \\
\hline & No & Observed & 37 & 54 & 13 & 5 & 2 & \multirow{2}{*}{111} & & \\
\hline & Total & 49 & 64 & 16 & 8 & 3 & 140 & & & \\
\hline \multirow{3}{*}{$\begin{array}{l}\text { Upper } \\
\text { back }\end{array}$} & Yes & Observed & 10 & 10 & 2 & 2 & 1 & 25 & \multirow{3}{*}{1.516} & \multirow{2}{*}{$\mathrm{p}>0.05$} \\
\hline & No & Observed & 39 & 54 & 14 & 6 & 2 & & & \\
\hline & Total & 49 & 64 & 16 & 8 & 3 & 140 & 115 & & \\
\hline \multirow{3}{*}{$\begin{array}{l}\text { Lower } \\
\text { Back }\end{array}$} & Yes & Observed & 14 & 15 & 3 & 2 & 1 & 35 & \multirow{3}{*}{0.861} & \multirow{2}{*}{$\mathrm{p}>0.05$} \\
\hline & No & Observed & 35 & 49 & 13 & 6 & 2 & & & \\
\hline & Total & 49 & 64 & 16 & 8 & 3 & 140 & 105 & & \\
\hline \multirow{3}{*}{ Shoulder } & Yes & Observed & 21 & 20 & 4 & 2 & 0 & 47 & \multirow{3}{*}{4.356} & \multirow{2}{*}{$\mathrm{p}>0.05$} \\
\hline & No & Observed & 28 & 44 & 12 & 6 & 3 & & & \\
\hline & Total & 49 & 64 & 16 & 8 & & 140 & 93 & & \\
\hline \multirow{3}{*}{ Elbows } & Yes & Observed & 7 & 5 & 1 & 1 & 0 & 14 & \multirow{3}{*}{1.979} & \multirow{2}{*}{$p>0.05$} \\
\hline & No & Observed & 42 & 59 & 15 & 7 & 3 & & & \\
\hline & Total & 49 & 64 & 16 & 8 & & 140 & 126 & & \\
\hline \multirow{3}{*}{$\begin{array}{l}\text { Wrists/ } \\
\text { hands }\end{array}$} & Yes & Observed & 5 & 4 & 1 & 0 & 0 & 10 & \multirow{3}{*}{1.635} & \multirow{2}{*}{$\mathrm{p}<0.05$} \\
\hline & No & Observed & 44 & 60 & 15 & 8 & 3 & & & \\
\hline & Total & 49 & 64 & 16 & 8 & & 140 & 130 & & \\
\hline \multirow{3}{*}{ Hips/ } & Yes & Observed & 7 & 6 & 0 & 0 & 0 & 13 & \multirow{3}{*}{4.219} & \multirow{2}{*}{$\mathrm{p}>0.05$} \\
\hline & No & Observed & 42 & 58 & 16 & 8 & 3 & \multirow{2}{*}{127} & & \\
\hline & Total & 49 & 64 & 16 & 8 & & 140 & & & \\
\hline \multirow{3}{*}{ Knees } & Yes & Observed & 20 & 34 & 7 & 3 & 0 & 64 & \multirow{3}{*}{4.659} & \multirow{2}{*}{$\mathrm{p}>0.05$} \\
\hline & No & Observed & 29 & 30 & 9 & 5 & 3 & & & \\
\hline & Total & 49 & 64 & 16 & 8 & & 140 & 76 & & \\
\hline & Yes & Observed & 12 & 13 & 3 & 3 & 0 & 31 & & $n>0,05$ \\
\hline Ankles/ & No & Observed & 37 & 51 & 13 & 5 & 3 & & 2.335 & $p>0.03$ \\
\hline & Total & 49 & 64 & 16 & 8 & & 140 & 109 & & \\
\hline
\end{tabular}

Wrist pain shows statistically significant association with computer usage $(\mathrm{p}<0.05)$. Inappropriate support and positioning of hands while using computers may be the reason for wrist pain. 
Rajarajeswary TR et.al., Musculoskeletal disorders in high school teachers of Tirur educational district-A cross sectional study

\section{Association between mode of transport and Musculoskeletal disorders}

Table No.9 Mode of transport and musculoskeletal disorders

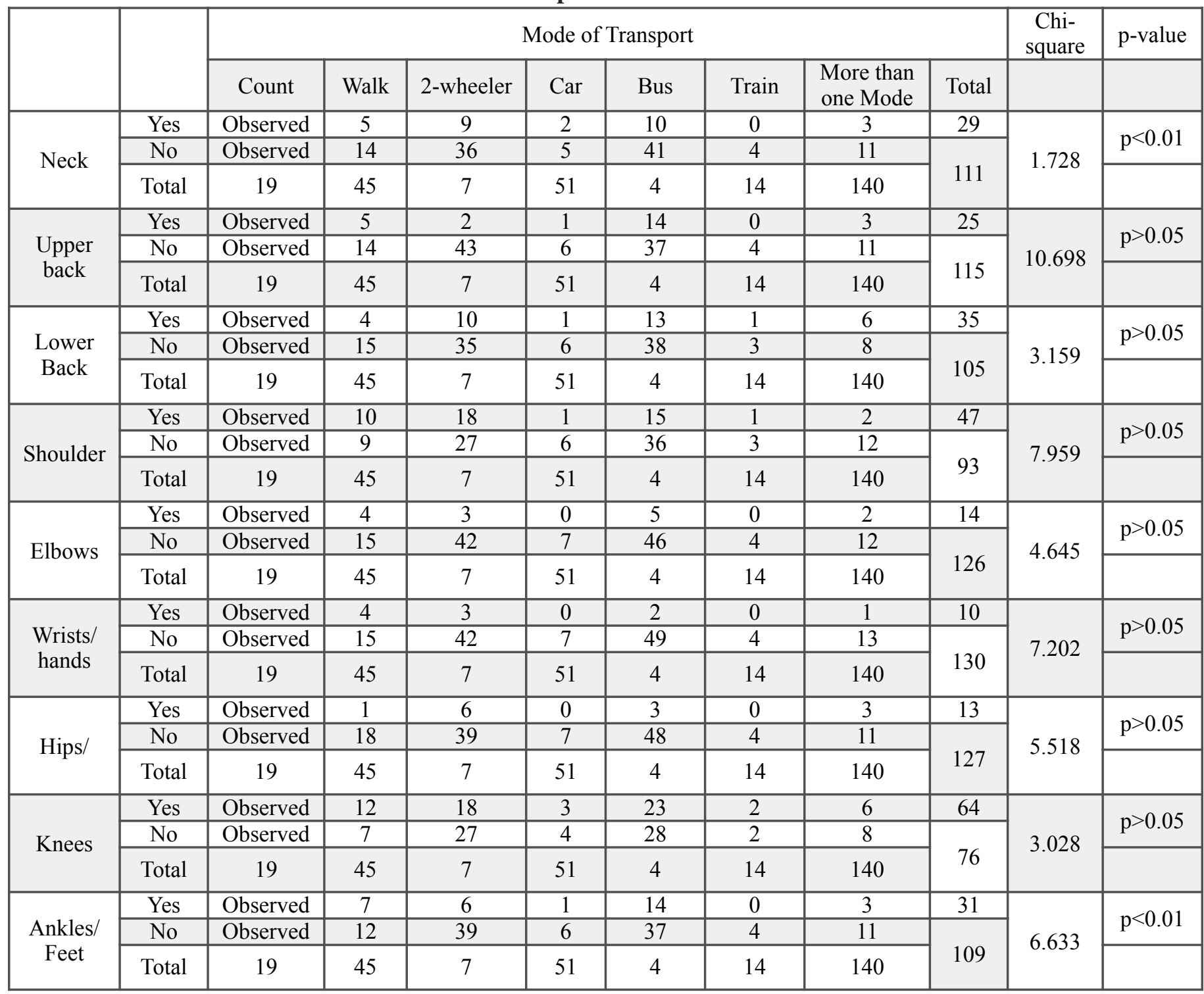

In the current study statistically significant association is shown by neck pain and ankle joint pain with mode of transport ( $<<0.01) .28 .6 \%$ who reported neck pain travel by the car, $20 \%$ by two wheeler and $19.3 \%$ by bus. It may be due to inappropriate upper back support. In the case of ankle joint pain $27.5 \%$ travel by bus and $36.8 \%$ travel by walk. It may be due to the unscientific construction of steps of bus.

9.Association between distance travelled to work place and musculoskeletal disorders Table No.10 Association of distance travelled to work place and musculoskeletal disorders

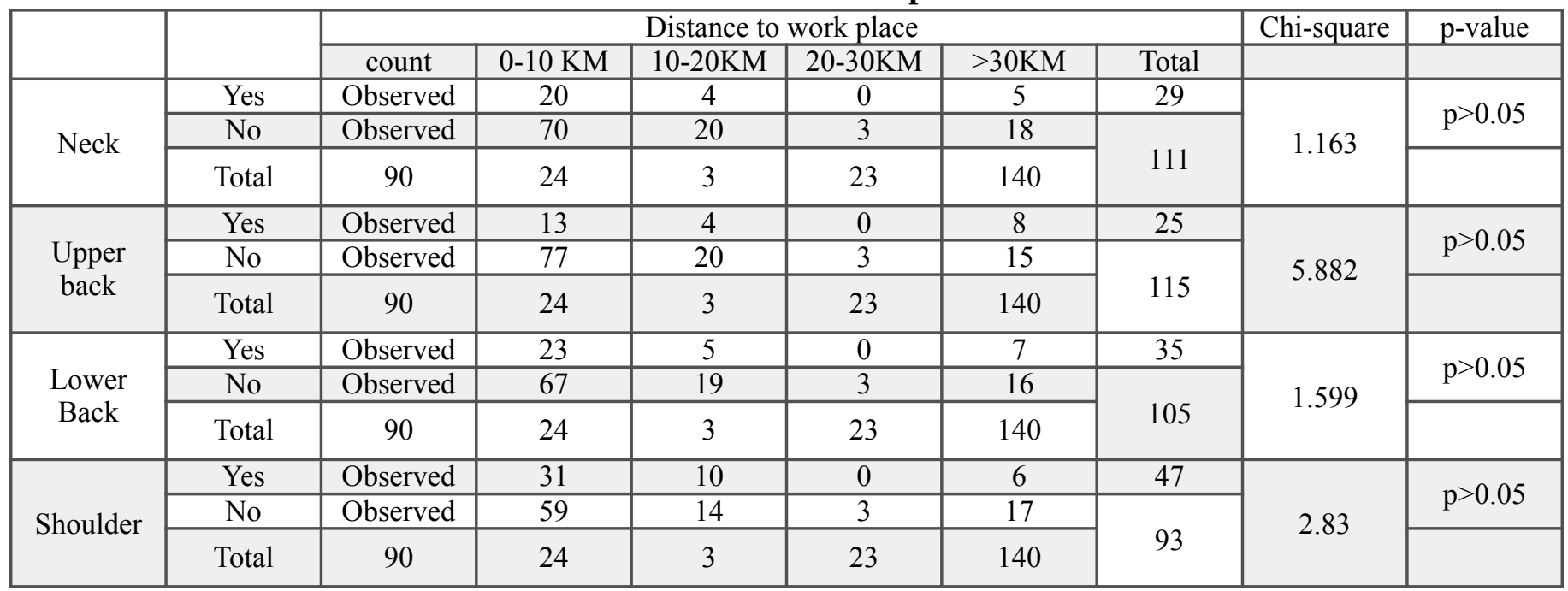


International Journal of Ayurvedic Medicine, Vol 11 (1), 98-107

\begin{tabular}{|c|c|c|c|c|c|c|c|c|c|}
\hline \multirow{3}{*}{ Elbows } & Yes & Observed & 8 & 5 & 0 & 1 & 14 & \multirow{3}{*}{4.403} & \multirow{2}{*}{$\mathrm{p}>0.05$} \\
\hline & No & Observed & 82 & 19 & 3 & 22 & \multirow[b]{2}{*}{126} & & \\
\hline & Total & 90 & 24 & 3 & 23 & 140 & & & \\
\hline \multirow{3}{*}{$\begin{array}{l}\text { Wrists/ } \\
\text { hands }\end{array}$} & Yes & Observed & 9 & 0 & 0 & 1 & 10 & \multirow{3}{*}{3.456} & \multirow{2}{*}{$\mathrm{p}<0.05$} \\
\hline & No & Observed & 81 & 24 & 3 & 22 & \multirow{2}{*}{130} & & \\
\hline & Total & 90 & 24 & 3 & 23 & 140 & & & \\
\hline \multirow{3}{*}{ Hips/ } & Yes & Observed & 10 & 3 & 0 & 0 & 13 & \multirow{3}{*}{3.312} & \multirow{2}{*}{$\mathrm{p}>0.05$} \\
\hline & No & Observed & 80 & 21 & 3 & 23 & \multirow{2}{*}{127} & & \\
\hline & Total & 90 & 24 & 3 & 23 & 140 & & & \\
\hline \multirow{3}{*}{ Knees } & Yes & Observed & 38 & 14 & 1 & 11 & 64 & \multirow{3}{*}{2.209} & \multirow{2}{*}{$\mathrm{p}>0.05$} \\
\hline & No & Observed & 52 & 10 & 2 & 12 & \multirow{2}{*}{76} & & \\
\hline & Total & 90 & 24 & 3 & 23 & 140 & & & \\
\hline \multirow{3}{*}{$\begin{array}{c}\text { Ankles/ } \\
\text { Feet }\end{array}$} & Yes & Observed & 21 & 5 & 1 & 4 & 31 & \multirow{3}{*}{0.617} & \multirow{2}{*}{$\mathrm{p}>0.05$} \\
\hline & No & Observed & 69 & 19 & 2 & 19 & \multirow{2}{*}{109} & & \\
\hline & Total & 90 & 24 & 3 & 23 & 140 & & & \\
\hline
\end{tabular}

Wrist pain shows statistically significant association with distance travelled $(\mathrm{p}<0.05)$. In the case of wrist pain $10 \%$ travel within $10 \mathrm{~km}$, only $4.3 \%$ travel more than $30 \mathrm{~km}$. Musculoskeletal disorders in other body sites shows no significant association with distance travelled.

\section{Overtime work and musculoskeletal disorders}

Neck pain shows statistically significant relation with overtime work $(\mathrm{p}<0.01)$. Wrist pain and ankle joint pain shows a positive association with overtime work $(\mathrm{p}<0.05)$. These findings are consistent with other studies. Prolonged standing in an inappropriate way for several hours inside the class room resulted in musculoskeletal pain among teachers. Lifting of hands and head during writing in the black board may be the causative factor for neck pain and also pain in hands and joints of hands. Teachers involved to a considerable physical load remaining in the orthostatic position up to $95 \%$ of activities, with varied levels of flexion of the backbone resulted in several types of physical inability.(10)

Table No.11 Association of overtime and musculoskeletal disorders

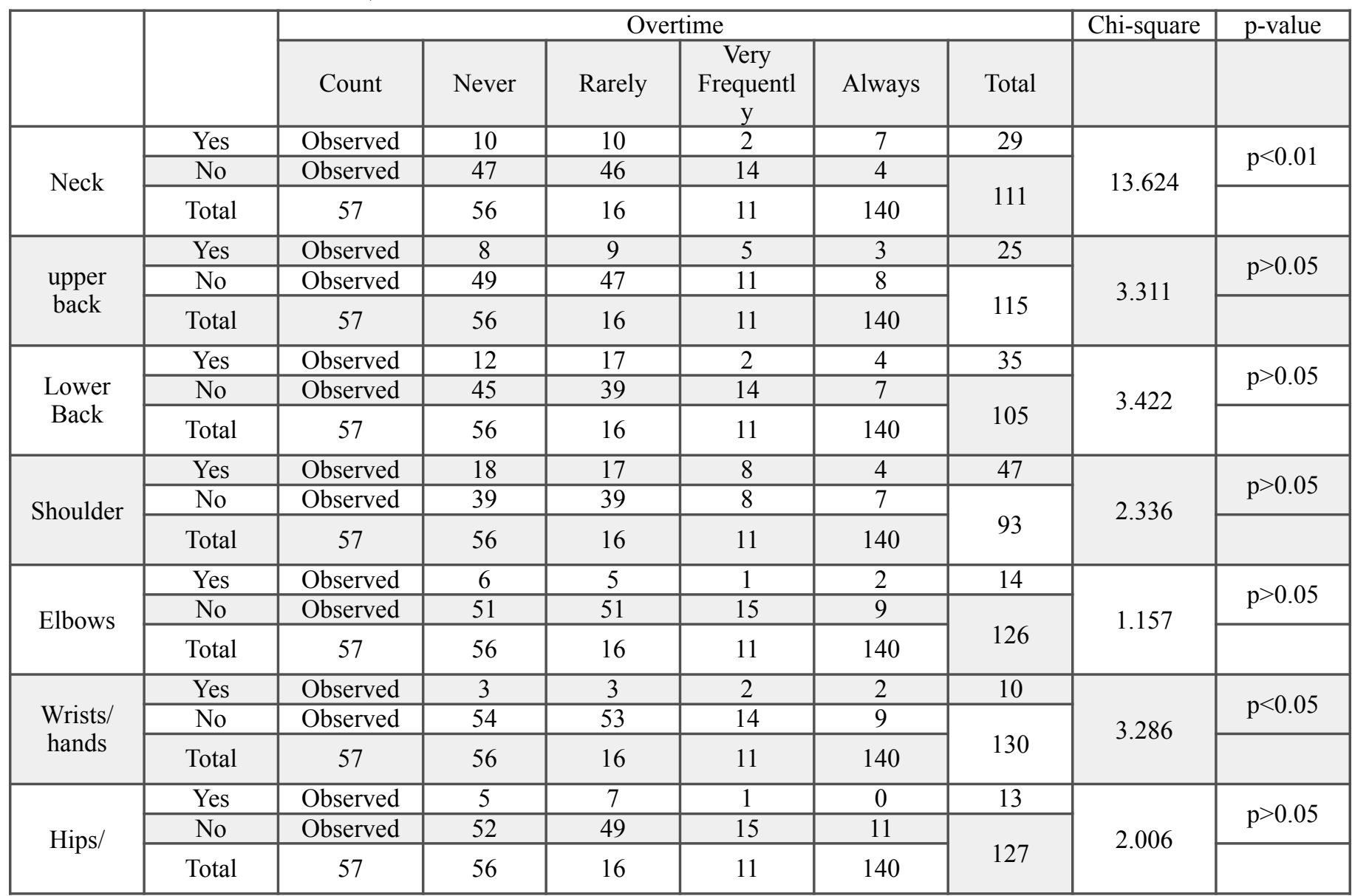


Rajarajeswary TR et.al., Musculoskeletal disorders in high school teachers of Tirur educational district-A cross sectional study

\begin{tabular}{|c|c|c|c|c|c|c|c|c|c|}
\hline \multirow{5}{*}{ Knees } & \multirow{2}{*}{ Yes } & Observed & 26 & 22 & 9 & 7 & 64 & \multirow{5}{*}{3.072} & \multirow{4}{*}{$\mathrm{p}>0.05$} \\
\hline & & Percentage & $45.6 \%$ & $39.3 \%$ & $56.3 \%$ & $63.6 \%$ & $45.7 \%$ & & \\
\hline & \multirow{2}{*}{ No } & Observed & 31 & 34 & 7 & 4 & 76 & & \\
\hline & & Percentage & $54.4 \%$ & $60.7 \%$ & $43.8 \%$ & $36.4 \%$ & \multirow{2}{*}{$54.3 \%$} & & \\
\hline & Total & 57 & 56 & 16 & 11 & 140 & & & \\
\hline \multirow{5}{*}{$\begin{array}{c}\text { Ankles/ } \\
\text { Feet }\end{array}$} & \multirow{2}{*}{ Yes } & Observed & 10 & 11 & 4 & 6 & 109 & \multirow{5}{*}{7.677} & \multirow{4}{*}{$\mathrm{p}<0.05$} \\
\hline & & Percentage & $17.5 \%$ & $19.6 \%$ & $25 \%$ & $54.5 \%$ & $22.1 \%$ & & \\
\hline & \multirow{2}{*}{ No } & Observed & 47 & 45 & 12 & 5 & 31 & & \\
\hline & & Percentage & $82.5 \%$ & $80.4 \%$ & $75 \%$ & $45.5 \%$ & \multirow{2}{*}{$77.9 \%$} & & \\
\hline & Total & 57 & 56 & 16 & 11 & 140 & & & \\
\hline
\end{tabular}

\section{Conclusion}

The present study was an attempt to assess the prevalence of musculoskeletal disorders among high school teachers of Tirur educational District.

Hypotheses formulated by the cross sectional analysis of high school teachers of Tirur educational district are:

1. Among high school teachers neck pain and ankle pain are associated with age.

2. Among high school teachers wrist pain and ankle pain are associated with female gender.

1. Among high school teachers wrist pain and hip pain is associated with years of teaching.

2. Among high school teachers neck pain ,wrist pain and ankle pain is associated with overtime work

Limitation - Study conducted only in one educational district among 140 teachers which may under estimate the prevalence.

Recommendations - A comparative study can be done including private school teachers. An observational study can be conducted in all Kerala Basis.

\section{References}

1. Park K. Park's text book of preventive and social medicine. 24th edit. Jabalapur: M/S Bhanot publisher; $2017.14 \mathrm{p}$

2. Vidya Ratan, Hand book of preventive and social medicine, 9th edition, NewDelhi: Jaypee Brother's Medical publishers (p) Ltd; 2010.1p

3. Suryakantha A.H Community medicine with recent advances 3 rd ed. jaypee brothers medical publishers (p) Ltd 2014.6p.
4. Erick P, Smith D. Musculoskeletal disorder risk factors in the teaching profession: a critical review. OA Musculoskeletal Medicine 2013 Dec $01 ; 1(3): 29$. Available from http:// www.oapublishinglondon.com/images/article/pdf/ 1399319378.pdf

5. Hildebrant V H, Bongers PM,Van Dijk FJH Kemper,H.C.G and J.Dul(2001) Dutch Musculoskeletal questionnaire:description and basic qualities Ergonomics,44(12), 1038-1055. Available from ergo.human.cornell.edu/studentdownload/ DEA4700pdfs/DMQ.pdfs

6. Erick P, Smith D. Musculoskeletal disorder risk factors in the teaching profession: a critical review. OA Musculoskeletal Medicine 2013 Dec 01;1(3):29. Available from http:// www.oapublishinglondon.com/images/article/pdf/ 1399319378.pdf

7. Chong EY, Chan AH. Subjective health complaints of teachers from primary and secondary schools in Hong kong. Int J occup Saf Ergon. 2010;16(1):23-29. Available from https:// www.ncbi.nlm.nih.gov>pubmed

8. Mariammal T, Amrita Jaisheeba A,Sornaraj R.Occupation influenced physical illness observed among the teachers of Thoothukudi town. Int.J.PharmTech Res.2012,4(3): 1274-1278. Available from sphinxsai.com $>$ pharm $>$ pdfpharm

9. ibid .

10. Mesaria S. and Jaiswal N. musculoskeletal disorders among teachers residing in various nations: A review. Research journal of recent sciences.4; 23-27. Available from www.isca.in. 